\title{
Fast and accurate boundary variation method for multilayered diffraction optics
}

\author{
L. C. Wilcox \\ Division of Applied Mathematics, Brown University, Providence, Rhode Island 02912 \\ P. G. Dinesen \\ Kaleido Technology Aps, Ryttermarken 15, DK-3520 Farum, Denmark \\ J. S. Hesthaven \\ Division of Applied Mathematics, Brown University, Providence, Rhode Island 02912
}

\begin{abstract}
Received October 29, 2003; revised manuscript received December 12, 2003; accepted December 17, 2003
A boundary variation method for the forward modeling of multilayered diffraction optics is presented. The approach permits fast and high-order accurate modeling of periodic transmission optics consisting of an arbitrary number of materials and interfaces of general shape subject to plane-wave illumination or, by solving a sequence of problems, illumination by beams. The key elements of the algorithm are discussed, as are details of an efficient implementation. Numerous comparisons with exact solutions and highly accurate direct solutions confirm the accuracy, the versatility, and the efficiency of the proposed method. (C) 2004 Optical Society of America
\end{abstract}

OCIS codes: $000.4430,050.1970,090.1970,230.1950,230.4170$.

\section{INTRODUCTION}

The ability to accurately and efficiently model multilayered diffraction-dominated optics has numerous applications, e.g., modeling of integrated transmission optics and the design and analysis of Bragg mirrors and integrated cavities. Such methods would also permit the modeling of random variations in design due to manufacturing limitations as well as serve as fast forward solvers in inversion as part of a quality control process.

For binary periodic structures the rigorous coupledwave analysis has been widely used to model such optical elements during the last decade. ${ }^{1}$ However, the need to analyze structures with aperiodic nonbinary features of finite extent and beam illumination requires that alternatives be sought. This has led to the development of several alternative approaches, e.g., finite-element, ${ }^{2}$ boundary element, ${ }^{3}$ finite-difference time-domain, ${ }^{4}$ and spectral collocation. $^{5-7}$ The latter two methods both compute a direct solution of the time-domain vectorial Maxwell equations and are very general, as they are applicable to a wide variety of geometries and physical settings. As the need to model problems of realistic size and complexity becomes more pressing, the memory and computational time requirements of such direct volume methods quickly become a limiting factor not only for the design process but also for the analyses of particular structures.

In this paper we take a different approach, building on the boundary variation method. ${ }^{8-10}$ This method is based on the observation that solutions to electromagnetic diffraction by a periodic structure depend analyti- cally on a variation of the interface. In other words, diffraction from a smooth grating can be determined from knowledge of reflection and refraction at a plane interface. With this result, fast and accurate high-order perturbation schemes for finite-size perturbations have been developed for modeling of two- and three-dimensional metallic and transmission gratings ${ }^{8-10}$ illuminated by plane waves. These methods were subsequently extended to include problems illuminated by guided waves ${ }^{11,12}$ and verified extensively by comparisons with direct high-order solution of Maxwell equations.

We continue the development of these methods by demonstrating their use in an iterative approach to accurately and efficiently model multilayered optics, e.g., transmission optics, where it is essential to accurately account for the internal reflections. To make this feasible, we discuss in some detail the implementation, which relies on properties of the scattering process to make it efficient. The proposed algorithm is shown to perform well, and the results agree very well with directly computed reference solutions, albeit obtained at a dramatically reduced computational cost.

What remains of this paper is organized as follows. In Section 2 we discuss the basic setup and the essential details of the formulation. This sets the stage for Section 3, where we summarize the boundary variation method, first for a single interface and subsequently for the general multiple-interface problem. Key elements of an efficient implementation are also discussed here. In Section 4 we offer a number of test cases to illustrate the effi- 
ciency and capability of the proposed approach, and Section 5 concludes with a few remarks and outlines future work.

\section{PROBLEM SETUP AND MODEL}

As illustrated in Fig. 1, we consider a two-dimensional situation in which a monochromatic plane wave

$$
\left(\begin{array}{c}
\mathbf{E}^{\text {inc }}(\mathbf{x}, t) \\
\mathbf{H}^{\text {inc }}(\mathbf{x}, t)
\end{array}\right)=\left(\begin{array}{c}
\mathbf{A}_{E} \\
\mathbf{A}_{H}
\end{array}\right) \exp \left[i\left(\mathbf{k}^{\text {inc }} \cdot \mathbf{x}-\omega t\right)\right]
$$

propagating in the homogeneous region $\Omega^{0}$, impinges on a structure of multilayered nonmagnetic homogeneous regions $\Omega^{1}, \ldots, \Omega^{N}$, separated by the smooth periodic interfaces $\Gamma^{1}, \ldots, \Gamma^{N}$, described by the functions $f^{1}(x), \ldots, f^{N}(x)$ with periods of length $L^{1}, \ldots, L^{N}$, respectively. For each region $\Omega^{j}$ we have the associated permittivities $\varepsilon^{j}$. Furthermore, we have introduced the complex amplitudes $\mathbf{A}_{E}$ and $\mathbf{A}_{H}$ for the electric and magnetic fields, respectively, while $\mathbf{k}^{\text {inc }}$ signifies the wavevector of the incoming field restricted to propagate in the $(x, y)$ plane, i.e., $\mathbf{k}^{\text {inc }}$ $=\left(k_{x}^{\text {inc }}, k_{y}^{\text {inc }}\right)$. Recall that

$$
\mathbf{k}^{\text {inc }}=\frac{2 \pi}{\lambda_{v}} \sqrt{\varepsilon^{0}} \hat{\mathbf{k}}^{\text {inc }}, \quad \omega=2 \pi \frac{c_{v}}{\lambda_{v}} .
$$

Without loss of generality we can normalize length and time such that the vacuum wavelength is $\lambda_{v}=1$ and the vacuum speed of light is $c_{v}=1$.

The illumination of the structure generates the fields $\left(\mathbf{E}^{j}, \mathbf{H}^{j}\right)$ in the region $\Omega^{j}$ for all $j \in\{0,1, \ldots, N\}$. These fields satisfy the time-harmonic Maxwell equations

$$
\nabla \times \mathbf{E}^{j}=i \omega \mathbf{H}^{j}, \quad \nabla \times \mathbf{H}^{j}=-i \omega \varepsilon^{j} \mathbf{E}^{j}
$$

under the constraint that the fields are solenoidal, i.e.,

$$
\nabla \cdot \mathbf{E}^{j}=\nabla \cdot \mathbf{H}^{j}=0, \quad \forall j \in\{0,1, \ldots, N\} .
$$

In $\Omega^{0}$ the total field is given as $\left(\mathbf{E}^{\text {inc }}, \mathbf{H}^{\text {inc }}\right)+\left(\mathbf{E}^{0}, \mathbf{H}^{0}\right)$; i.e., in this region $\left(\mathbf{E}^{0}, \mathbf{H}^{0}\right)$ represents the scattered field, and $\left(\mathbf{E}^{j}, \mathbf{H}^{j}\right)$ signifies the total fields elsewhere.

The fields in the homogeneous regions $\Omega^{j-1}$ and $\Omega^{j}$ are connected through the boundary conditions along $\Gamma^{j}$ for all $j \in\{1, \ldots, N\}$. In particular, along the interface $\Gamma^{j}$, endowed with an outward-pointing normal vector $\hat{\mathbf{n}}^{j}$, separating the two dielectric regions $\Omega^{j-1}$ and $\Omega^{j}$, continuity of the tangential field components requires that

$$
\hat{\mathbf{n}}^{j} \times\left(\begin{array}{c}
\mathbf{E}^{j-1}+\delta_{j-1,0} \mathbf{E}^{\text {inc }} \\
\mathbf{H}^{j-1}+\delta_{j-1,0} \mathbf{H}^{\text {inc }}
\end{array}\right)=\hat{\mathbf{n}}^{j} \times\left(\begin{array}{c}
\mathbf{E}^{j} \\
\mathbf{H}^{j}
\end{array}\right),
$$

where $\delta_{i, j}$ is the Kronecker delta. In the special case where $\Gamma^{j}$ is assumed to be a perfect conductor, this condition degenerates to a Dirichlet condition on the electric field as

$$
\hat{\mathbf{n}}^{j} \times \mathbf{E}^{j-1}=-\hat{\mathbf{n}}^{j} \times \delta_{j-1,0} \mathbf{E}^{\mathrm{inc}} .
$$

When one is solving the Maxwell equations, it is often advantageous to express the boundary condition on $\mathbf{H}^{j}$ through the condition on $\mathbf{E}^{j}$ and the equations themselves as a Neumann condition

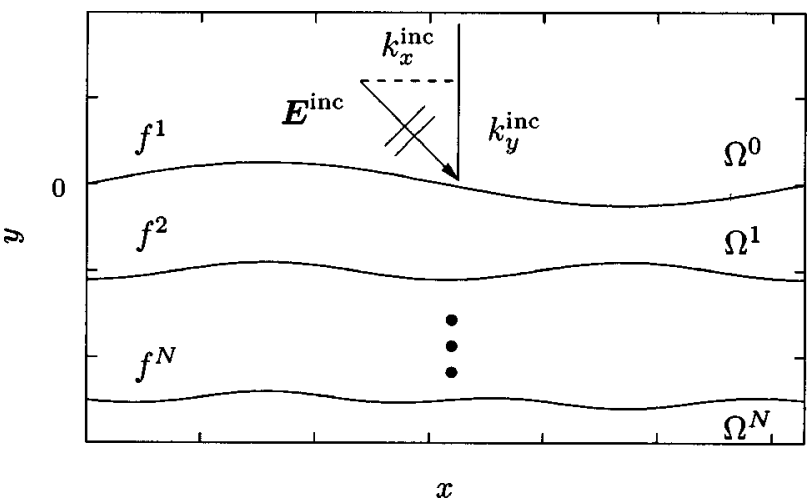

Fig. 1. Generic setup for scattering by a problem with multiple interfaces.

$$
\begin{aligned}
\hat{\mathbf{n}}^{j} \times \nabla \times\left(\mathbf{H}^{j-1}+\delta_{j-1,0} \mathbf{H}^{\text {inc }}\right) & \\
& =-\hat{\mathbf{n}}^{j} \cdot \nabla\left(\mathbf{H}^{j-1}+\delta_{j-1,0} \mathbf{H}^{\text {inc }}\right) \\
& =-\frac{\partial\left(\mathbf{H}^{j-1}+\delta_{j-1,0} \mathbf{H}^{\text {inc }}\right)}{\partial \hat{\mathbf{n}}^{j}}=0
\end{aligned}
$$

along the perfectly conducting interface $\Gamma^{j}$.

To simplify matters further, we restrict ourselves to problems where $\Omega^{j}$ can be considered homogeneous and lossless and where the incoming fields are two dimensional and either TE or TM polarized. This implies that the local solutions to the scattering/penetration problems satisfy the homogeneous Helmholtz equation

$$
\Delta u^{j}+\left|k^{j}\right|^{2} u^{j}=0, \quad j=0, \ldots, N,
$$

where $u^{j}=E_{z}^{j}$ in the case of TE-polarized illumination and $u^{j}=H_{z}^{j}$ for a TM-polarized incoming wave. Furthermore, $\left|k^{j}\right|=2 \pi \sqrt{\varepsilon^{j}}$ represents the magnitude of the local wave vector under the normalization discussed above.

This system of Helmholtz equations [Eq. (1)], must be solved subject to the conditions that

$$
u^{j-1}\left(x, f^{j}(x)\right)-u^{j}\left(x, f^{j}(x)\right)=-\delta_{j-1,0} u^{\text {inc }}\left(x, f^{j}(x)\right),
$$

$$
\begin{aligned}
\frac{\partial}{\partial \hat{\mathbf{n}}^{j}} u^{j-1}\left(x, f^{j}(x)\right)-C_{j} \frac{\partial}{\partial \hat{\mathbf{n}}^{j}} u^{j}(x, & \left.f^{j}(x)\right) \\
& =-\frac{\partial}{\partial \hat{\mathbf{n}}^{j}} \delta_{j-1,0} u^{\mathrm{inc}}\left(x, f^{j}(x)\right)
\end{aligned}
$$

at a general dielectric interface. The constant $C_{j}$ takes the values

$$
C_{j}=\left\{\begin{array}{ll}
1, & \text { TE polarization } \\
\varepsilon^{j-1} / \varepsilon^{j}, & \text { TM polarization }
\end{array} .\right.
$$

In the case where $\Gamma^{j}$ represents a perfectly conducting object, the conditions are different for the two polarizations; i.e., we have a Dirichlet condition in the case of a TEpolarized wave, given by

$$
u^{j-1}\left(x, f^{j}(x)\right)=-\delta_{j-1,0} E_{z}^{\text {inc }}\left(x, f^{j}(x)\right),
$$

while we recover a Neumann condition on the tangential magnetic field as 


$$
\frac{\partial}{\partial \hat{\mathbf{n}}_{j}} u^{j-1}\left(x, f^{j}(x)\right)=-\frac{\partial}{\partial \hat{\mathbf{n}}_{j}} \delta_{j-1,0} H_{z}^{\mathrm{inc}}\left(x, f^{j}(x)\right)
$$

along the metallic interface in the case where the illuminating wave is TM polarized.

To complete the specification of the problem, we require that the solutions $u^{0}$ and $u^{N}$ be bounded at infinity and that the solutions consist of purely outgoing waves. The means by which we enforce these conditions are closely related to the computational approach chosen to solve the above system of coupled Helmholtz equations.

\section{BOUNDARY VARIATION METHOD}

As we solve the general multiple-interface problem through a sequence of single-interface problems, we begin by discussing the single-interface scheme in detail and subsequently consider the extension to the general case.

\section{A. Single-Interface Scheme}

Let us assume that we only have a single interface $\Gamma$, separating the two homogeneous regions $\Omega^{+}=\Omega^{0}$ and $\Omega^{-}=\Omega^{1}$, illuminated by a two-dimensional TE- or TMpolarized wave. We take the amplitude of the illuminating TE or TM wave to be 1 .

The interface $\Gamma$ is assumed to be $L$-periodic along $x$ and described by $f(x, L)$. Since the incident wave is a plane wave, the fields $u^{ \pm}(\mathbf{x})$ are endowed with a similar periodicity, i.e.,

$$
u^{ \pm}(x+L, y)=\exp \left(i k_{x}^{\text {inc }} L\right) u^{ \pm}(x, y) .
$$

Following Ref. 13, we shall fix the notation by introducing

$$
K=\frac{2 \pi}{L}, \quad \alpha_{n}=k_{x}^{\text {inc }}+n K, \quad \alpha_{n}^{2}+\left(\beta_{n}^{ \pm}\right)^{2}=\left|k^{ \pm}\right|^{2} .
$$

Here $K$ simply reflects the wave number associated with the periodicity, $\alpha_{n}$ represents the Bragg condition for lattice refraction/reflection, while the last condition expresses energy conservation. To determine $\beta_{n}^{ \pm}$in accordance with the orientation of the problem (see Fig. 1), we shall use the notation that for the propagating waves $\beta_{n}^{ \pm}$ $\leqslant 0$ as the incoming wave propagates in the negative $y$ direction. To ensure that the waves are bounded at infinity, we let $\operatorname{Im}\left(\beta_{n}^{ \pm}\right)<0$ for the evanescent waves. Clearly there can be only a finite number of propagating modes since, for $n$ sufficiently large, $\beta_{n}^{ \pm}$becomes purely imaginary.

Away from the interface $\Gamma$, we now express the solution $u^{ \pm}(x, y)$ as a Rayleigh expansion:

$$
u^{ \pm}(x, y)=\sum_{n=-\infty}^{\infty} B_{n}^{ \pm} \exp \left[i\left(\alpha_{n} x \mp \beta_{n}^{ \pm} y\right)\right] .
$$

Conservation of energy implies that

$$
\sum_{n \in \Pi^{+}} \beta_{n}^{+}\left|B_{n}^{+}\right|^{2}+C_{0} \sum_{n \in \Pi^{-}} \beta_{n}^{-}\left|B_{n}^{-}\right|^{2}=k_{y}^{\text {inc }},
$$

where $\Pi^{ \pm}$represent the subset of $\beta_{n}^{ \pm}$corresponding to the propagating waves; i.e., $\beta_{n}^{ \pm} \leqslant 0$ is real. For purely metallic scattering the second part of the sum drops out.
In this setting the unknowns are the Rayleigh coefficients $B_{n}^{ \pm}$, which depend on the profile $f(x, L)$. We introduce a new profile,

$$
f_{\delta}(x)=\delta f(x, L)
$$

i.e., $\delta=0$ corresponds to a flat horizontal interface, and $\delta=1$ represents the profile of interest.

The heart of the boundary variation method ${ }^{8,9}$ is the assumption that the Rayleigh coefficients $B_{n}^{ \pm}(\delta)$ can be expressed as a Taylor series in $\delta$,

$$
B_{n}^{ \pm}(\delta)=\left.\sum_{k=0}^{\infty} \frac{1}{k !} \frac{\mathrm{d}^{k} B_{n}^{ \pm}(\delta)}{\mathrm{d} \delta^{k}}\right|_{\delta=0} \delta^{k}=\sum_{k=0}^{\infty} d_{k, n} \delta^{k} ;
$$

i.e., we assume that $B_{n}^{ \pm}$, and hence $u^{ \pm}$, are analytic in the boundary variation $\delta$. The validity of this is by no means obvious but has nevertheless been established rigorously. ${ }^{14}$

To illustrate the way by which one can obtain $d_{k, n}$, we consider the simplest case in which $\Omega^{-}$is a perfectly conducting metallic object illuminated by a TE-polarized plane wave, i.e., the boundary condition is

$$
u^{+}\left(x, f_{\delta}(x)\right)=-\exp \left[i k_{x}^{\mathrm{inc}} x+i k_{y}^{\mathrm{inc}} \delta f(x, L)\right] .
$$

From the Rayleigh expansion itself, we have

$$
\left.\frac{1}{k !} \frac{\partial^{k} u^{+}}{\partial \delta^{k}}\right|_{\delta=0}=\sum_{n=-\infty}^{\infty} d_{k, n} \exp \left[i\left(\alpha_{n} x-\beta_{n}^{+} y\right)\right] .
$$

We can, however, using the boundary condition, also evaluate the variation of $u^{+}$with respect to $\delta$ at $y=0$ as

$$
\begin{aligned}
\left.\frac{1}{r !} \frac{\partial^{r} u^{+}}{\partial \delta^{r}}\right|_{y, \delta=0}= & -\left(i k_{y}^{\text {inc }}\right)^{r} \frac{f^{r}}{r !} \exp \left(i k_{x}^{\text {inc }} x\right) \\
& -\sum_{k=0}^{r-1} \frac{f^{r-k}}{(r-k) !} \frac{\partial^{r-k}}{\partial y^{r-k}}\left(\frac{1}{k !} \frac{\partial^{k} u^{+}}{\partial \delta^{k}}\right)_{y, \delta=0} .
\end{aligned}
$$

Let us introduce the Fourier expansion of the periodic interface $f(x, L)$, as well as powers of it, as

$$
\frac{[f(x, L)]^{r}}{r !}=\sum_{l=-r F}^{r F} C_{r, l} \exp (i K l x)
$$

Using Eq. (4) to evaluate the $y$ derivative of $(k !)^{-1}\left(\partial^{k} u^{+} / \partial \delta^{k}\right)$, we can, by combining Eqs. (4)-(6), recover an explicit forward recurrence for the unknown expansion coefficients $d_{k, n}$ of the Rayleigh coefficients in the form

$$
\begin{aligned}
d_{k, n}= & -\left(i k_{y}^{\mathrm{inc}}\right)^{k} C_{k, n} \\
& -\sum_{r=0}^{k-1} \sum_{q=\max [-r F, n-(k-r) F]}^{\min [r F, n+(k-r) F]} C_{k-r, n-q}\left(-i \beta_{q}\right)^{k-r} d_{r, q} .
\end{aligned}
$$

Hence, given all expansion coefficients $d_{s, t}$ and $C_{s, t}$ for $s$ $<k$ and $-s F \leqslant t \leqslant s F$, we can recover all expansion coefficients for $s=k$ by forward recurrence.

Albeit of a more complicated form, similar recurrences can be derived for TM-polarized illumination ${ }^{8}$ as well as 
for TE- and TM-polarized illumination of a general dielectric interface. ${ }^{9}$ Recurrences for illumination by guided waves are also known. ${ }^{11,12}$

Although this yields an approach for computing the Taylor expansion of the Rayleigh coefficients,

$$
B_{n}(\delta)=\sum_{k=0}^{D} d_{k, n} \delta^{k}
$$

it is generally not an easy matter to evaluate this expansion outside its circle of convergence. To partially overcome this, we express the Taylor series by its Padé approximant as

$$
[L / M]=\frac{a_{0}+a_{1} \delta+\cdots+a_{L} \delta^{L}}{1+b_{1} \delta+\cdots+b_{M} \delta^{M}},
$$

where the coefficients are found with standard means by requiring that its Taylor series be equivalent to the Taylor series $B_{n}(\delta)$ up to order $M+L+1 \leqslant D$. It is wellknown that Padé approximations have remarkably better convergence properties than Taylor series, ${ }^{15}$ and their use provides adequate convergence for the present investigation.

With this in place we can compute the Rayleigh coefficients of the global solution $u^{ \pm}(x, y)$ at different angles of incidence, controlled by $\mathbf{k}^{\text {inc }}$, using only knowledge of the Fourier representation of the interface $\Gamma$.

\section{B. Multiple-Interface Scheme}

Exploiting the linearity of the Helmholtz equation and considering a geometrical-optics series, we will use the single-interface scheme repeatedly to form the scheme for multiple interfaces. The approximate solution for the single-interface case is given as a plane- and evanescentwave expansion:

$$
u^{ \pm}(x, y)=\sum_{n=-p^{ \pm}-E_{e}}^{q^{ \pm}+E_{e}} B_{n}^{ \pm} \exp \left[i\left(\alpha_{n} x \mp \beta_{n}^{ \pm} y\right)\right],
$$

where $n \in\left\{-p^{ \pm}, \ldots, q^{ \pm}\right\}$is the set of waves for which $\beta_{n}^{ \pm}$ $\leqslant 0$ is real and $E_{e}$ is a nonnegative integer. The nonpropagating waves can be disregarded in the singleinterface case by setting $E_{e}=0$ if the focus is on the modeling of the far field. For other applications requiring detailed near-field information, these evanescent waves could be included by setting $E_{e}>0$. This will include the $2 E_{e}$ dominating evanescent waves for each interface.

In the multiple-interface scheme the solution for the first interface, $\Gamma^{1}$ is computed first. This is a sum of plane waves propagating away from the interface and evanescent waves [Eq. (7)]. The plane waves traveling away from the multilayer structure are not propagated any further. However, the plane waves traveling down into the multilayer structure are propagated to the next interface by updating the phase by a factor that depends on the diffraction order of the wave and the unperturbed vertical distance between the interfaces. We do not propagate the evanescent waves, thus disregarding optical tunneling. There is nothing in the method that prevents us from propagating the evanescent waves, but for our interest in transmission optics problems we have not seen the need. For problems such as waveguide coupling, where the accurate representation of the field inside the optics is of interest, propagation of the evanescent waves may need to be investigated. The singleinterface boundary variation method is subsequently used on each of these propagated waves at the new interface. As this process continues, a set of plane and evanescent waves in each region is computed, the sum of which gives the approximate solution in that region.

By conservation of energy there must be a finite number of directions in which the plane waves can travel within each region, i.e., the multiple-interface scheme evokes the single-interface scheme only a finite number of times. When the multiple-interface algorithm starts, the single-interface scheme is called a finite number of times for a wave of unit amplitude in all the possible planewave directions to build an efficiency table. When the single-interface scheme is needed, a lookup in the efficiency table based on the direction of the incident wave and the region that it lives in produces the efficiencies and the directions of the refracted and reflected waves. The amplitudes of the refracted and reflected waves are found by multiplying the efficiencies by the amplitude of the incident wave. Using the fact that there are a finite number of possible waves, the method can group waves to limit the computations. Subsection 3.C will make this need apparent in order to render the approach computationally efficient.

\section{Implementation}

Let us first consider the example of a double-interface structure (Fig. 2). The structure has a top interface $\Gamma^{1}$, which gives rise to three diffracted plane waves when illuminated. To keep this example simple, we take $E_{e}$ $=0$, disregarding the evanescent waves. The second interface $\Gamma^{2}$ is taken to be planar for simplicity. With the

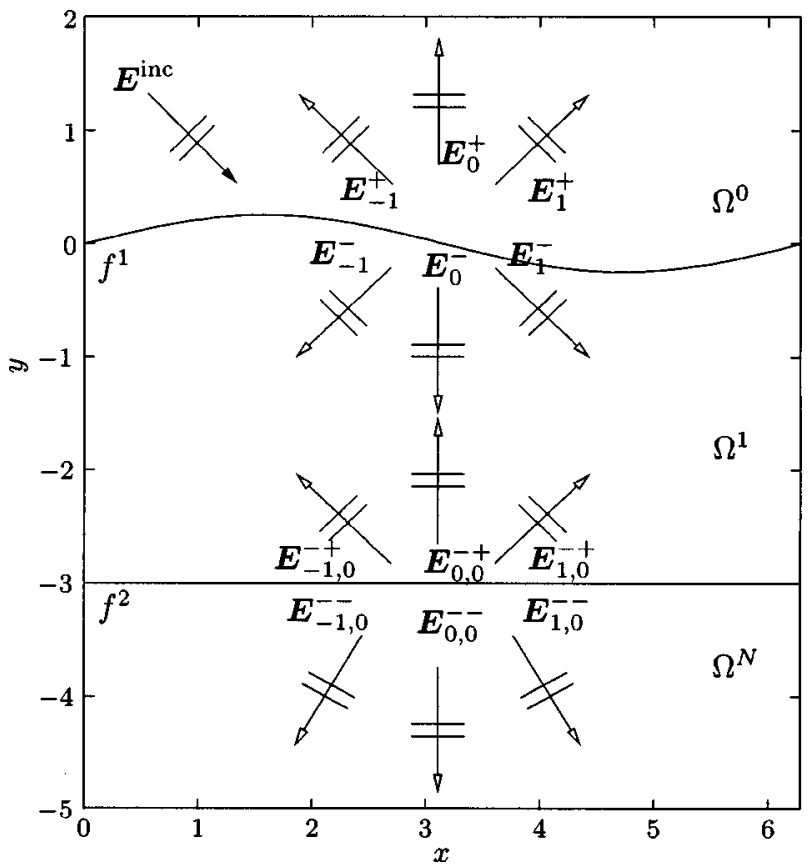

Fig. 2. Specific example to illustrate scheme for a problem with two interfaces. 
incident plane wave $\mathbf{E}^{\text {inc }}(\mathbf{x}, t)$ and the top interface $\Gamma^{1}$, the single-interface boundary variation method is used to compute the solution

$$
\mathbf{E}^{ \pm}(x, y)=\sum_{n=-1}^{1} \mathbf{E}_{n}^{ \pm},
$$

where

$$
\mathbf{E}_{n}^{ \pm}=B_{n}^{ \pm} \exp \left[i\left(\alpha_{n} x \mp \beta_{n}^{ \pm} y\right)\right], \quad \forall n \in\{-1,0,1\} .
$$

We assume that the boundary variation method emits a solution that is the summation of three plane waves for the region $\Omega^{0}$ and three plane waves for the region $\Omega^{1}$. To track the solutions, the implementation employs a solution set $S^{n}$ associated with each region $\Omega^{n}$. These are initialized to the empty set for $n \in\{0,1,2\}$. The solutions are added to their respective sets: $S^{0}$ $=S^{0} \cup\left\{\mathbf{E}_{n}^{+}\right\}_{n=-1}^{1}$ and $S^{1}=S^{1} \cup\left\{\mathbf{E}_{n}^{-}\right\}_{n=-1}^{1}$. The plane waves $\left\{\mathbf{E}_{n}^{+}\right\}_{n=-1}^{1}$ travel away from the interface and require no further consideration before evaluation of the far field. The remaining waves $\left\{\mathbf{E}_{n}^{-}\right\}_{n=-1}^{1}$ are traveling toward $\Gamma^{2}$, and we propagate these waves by simply updating the phase. For the wave $\exp \left(i \beta_{-1}^{-} d\right) \mathbf{E}_{-1}^{-}$, we need to solve the single-interface problem at $\Gamma^{2}$. However, as $\Gamma^{2}$ is a flat plane, we use Fresnel's equations to compute exactly the reflected and refracted waves, $\mathbf{E}_{-1,0}^{-+}$and $\mathbf{E}_{-1,0}^{--}$, respectively. The waves are added to the solution sets, so $S^{1}=S^{1} \cup\left\{\mathbf{E}_{-1,0}^{-+}\right\}$and $S^{2}=S^{2} \cup\left\{\mathbf{E}_{-1,0}^{--}\right\}$. The wave $\mathbf{E}_{-1,0}^{--}$is propagating in the negative $y$ direction away from the optical element and need not be considered further. However, $\mathbf{E}_{-1,0}^{-+}$is traveling toward $\Gamma^{1}$ and eventually interacts with $\Gamma^{1}$ from below; i.e., the interaction is computed by using the single-interface algorithm with the $-f^{1}(x)$ profile. This process of collecting the solution waves from the single-interface boundary condition and propagating the waves in $\Omega^{1}$ is accounting for multiple internal reflections and is continued as long as needed.

For the general multilayer problem with $N$ interfaces, we initialize our method with the region $\Omega^{0}$ and the incident wave $\mathbf{E}^{\text {inc }}$ by adding $\left(\mathbf{E}^{\text {inc }}, \Omega^{0}\right.$ ) to the wave set $W^{0}$. Here $W^{0}$ is the active set that contains the waves that need to be propagated to the next interface. The superscript on $W$ represents the number of iterations, or bounces, that the waves in the wave set have undergone. Let $T^{\mathrm{ttl}}$ be a positive integer that is the maximum number of bounces that the calculation is permitted to take. All of the solution sets are initialized to the empty set: $S^{1}$ $=\cdots=S^{N}=\emptyset$. Also, let $D^{l}=\sum_{j=0}^{l} d^{j}$, where $d^{j}$ is the unperturbed thickness of the layer $\Omega^{j}$. With this notation we must choose $d^{0}=d^{N}=0$.

To initialize the efficiency table, we first note that in any given layer the local Bragg condition is $\alpha=k_{x}^{\text {inc }}$ $+\sum_{j=1}^{N} K^{j} m_{j}$, where $K^{j}=(2 \pi) / L^{j}$ and $m_{j}$ is an integer for $j=1,2, \ldots, N$. Also, for each region $\Omega^{j}$ we have conservation of energy:

$$
\alpha^{2}+\beta^{2}=\left|k^{j}\right|^{2} .
$$

Recall that we propagate only the waves for which $\beta$ is real, i.e., there are a finite number of waves in each layer and we can find all possible directions that a wave will be traveling in each layer by using the Bragg conditions. The single-interface boundary variation code can be run for incident waves of unit magnitude for each of these possible directions in each layer, and the resulting efficiencies are stored in a separate hash table for each layer. The key to the hash table is the direction of the incident wave. In the case of an incident wave illumination of a flat plane interface, the computations are simplified by using Fresnel's equations ${ }^{16}$ instead of the single-interface boundary variation code.

Assume now that we are considering a wave set that has undergone $m$ bounces and is scheduled to undergo the next bounce. First, set $W^{m+1}=\emptyset$. For each element $w$ $=\left(\mathbf{E}, \Omega^{l}\right)$ from the set of active waves $W^{n}$, we have $\mathbf{E}$ $=A \exp [i(\alpha x+C \beta y)]$, where $C=-1$ if the wave is traveling in the positive $y$ direction and $C=1$ if the wave is traveling in the negative $y$ direction. Note that the wave $\mathbf{E}$ is going to interact with interface $f^{l^{*}}$, where $l^{*}$ $=\max (l, l+C)$. The wave $\mathbf{E}$ is propagated by updating the phase through multiplication by $\exp \left(i \beta d^{l}\right)$ and using the single-interface method for the interface $f^{l^{*}}+D^{l^{*}}$ with the initial wave $\mathbf{E} \exp \left(i \beta d^{l}\right)$. This yields the solution

$$
u^{ \pm}(x, y)=\sum_{n=-p^{ \pm}-E_{e}}^{q^{ \pm}+E_{e}} \mathbf{E}_{n}^{ \pm},
$$

where

$$
\begin{aligned}
& \mathbf{E}_{n}^{ \pm}=B_{n}^{ \pm} \exp \left[i\left(\alpha_{n} x \mp \beta_{n}^{ \pm} y\right)\right] \\
& \forall n \in\left\{-E_{e}-p^{ \pm},-E_{e}-p^{ \pm}+1, \ldots, q^{ \pm}+E_{e}\right\} .
\end{aligned}
$$

Each wave is added to a set corresponding to each layer, i.e., $\quad S^{l}=S^{l} \cup\left\{\exp \left(-i C \beta D^{l^{*}}\right) \mathbf{E}_{n}^{+}\right\}_{n=-p^{+}-E_{e}}^{q^{+}+E_{e}}$ and $S^{l+C}$ $=S^{l+C} \cup\left\{\exp \left(-i C \beta D^{l^{*}}\right) \mathbf{E}_{n}^{-}\right\}_{n=-p^{-}-E_{e}}^{q^{-}+E_{e}}$.

Next, we determine if we should add the newly computed waves to the set $W^{m+1}$ of waves to be propagated in the $(m+1)$ th bounce. If $m=T^{\mathrm{ttl}}$, then the waves' "time to live" is up and none of the waves is added to $W^{m+1}$. Alternatively, if a particular amplitude is below a given threshold $w_{\epsilon}$, we can decide not to propagate this wave any further. Furthermore, if $l+C \in\{1,2, \ldots, N\}$, then the waves $\left(\left\{\mathbf{E}_{n}^{-}\right\}_{n=-p^{-}}^{q^{-}}, \Omega^{-}\right)$are added to the set $W^{m+1}$. If $C \neq 1 \vee l \neq 0$, then the waves $\left(\left\{\mathbf{E}_{n}^{+}\right\}_{n=-p^{+}}^{q^{+}}\right.$, $\Omega^{+}$) are added to the set $W^{m+1}$. Note that the evanescent waves are not added to $W^{m+1}$. When waves are added to the set $W^{m+1}$, it is first checked whether a wave with the same direction and region is already in $W^{m+1}$. If so, then the amplitude of the wave is added to the amplitude of the wave already in the set. If there is no wave with the same direction in the same region, then the wave is simply added to $W^{m+1}$. Owing to conservation of energy, there are only a maximum of $P$ waves independent of $m$ in $W^{m+1}$. Without this accounting for multiple waves at a similar direction of propagation, the number of active waves would grow exponentially, rendering the computational work prohibitive.

The end of bounce $m$ is reached when all the waves in $W^{m}$ have been propagated and their children have been added to $W^{m+1}$ according to the above restrictions. Note that the computation stops when $W^{m+1}=0$. To get the approximate multilayer solution in the region $\Omega^{l}$, we sum up the elements in $S^{l}$. 


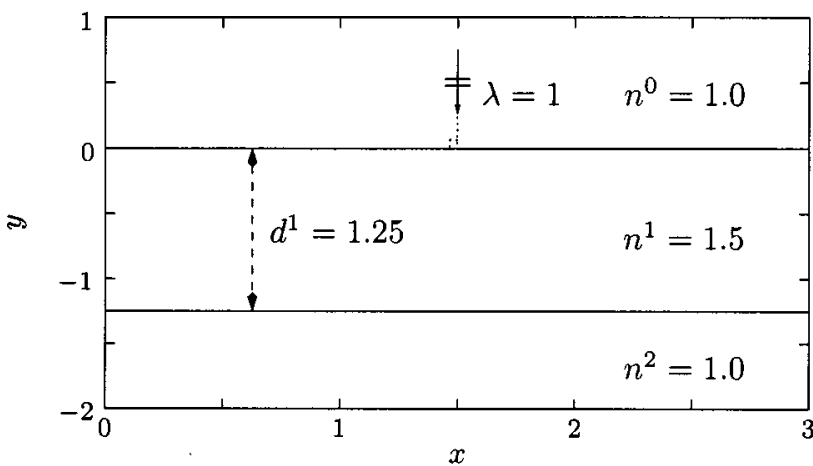

(a)

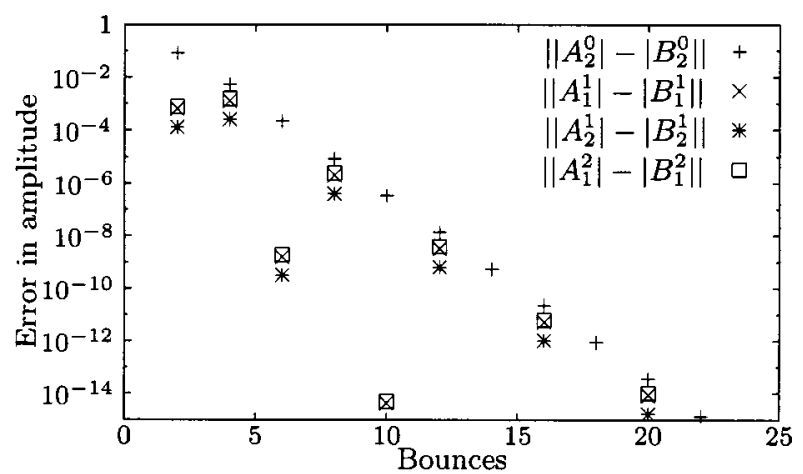

(b)

Fig. 3. (a) Problem setup for the test with one plane layer, (b) decay of error in field amplitudes as a function of internal reflections or bounces.

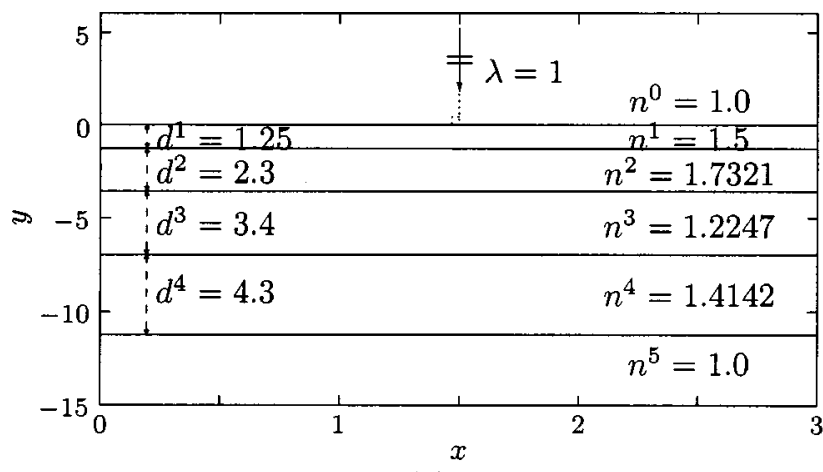

(a)

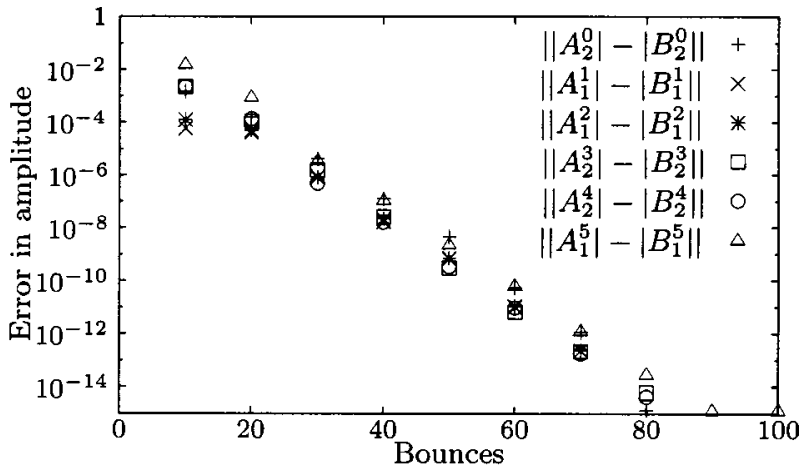

(b)

Fig. 4. (a) Problem setup for the test with a stack of plane layers, (b) decay of error in field amplitudes as a function of internal reflections or bounces.

It is possible to use the $L_{2}$ difference in solutions of different $T^{\text {ttl }}$ s at a few given $y$ values near the interfaces to evaluate the convergence as a measure of accuracy. In each region $\Omega^{j}$, there is a solution set $S^{j}$ that holds a finite number of waves, e.g., $s^{j}$. We consider the error $\epsilon$ in energy by the relation for the scattering efficiencies:

$$
\epsilon=\sum_{l=1}^{s^{0}} e_{l}^{0}+\frac{\varepsilon^{0}}{\varepsilon^{N}} \sum_{l=1}^{s^{N}} e_{l}^{N}-1
$$

where $e_{l}^{j}=\beta_{l}^{j}\left|B_{l}^{j}\right|^{2} / \beta^{\text {inc }}$ is the efficiency and $B_{l}^{j} \exp \left[i\left(\alpha_{l}^{j} x\right.\right.$ $\left.\left.+C_{l}^{j} \beta_{l}^{j} y\right)\right]$ is a wave in $S^{j}$. Note that $S^{0}$ does not include the incident wave and $C_{l}^{j}=-1,1$ denotes the direction in which the wave is traveling.

It is worthwhile emphasizing that a small energy deficiency $\epsilon$ does not imply convergence to the correct solution but simply checks self-consistency.

\section{VERIFICATION AND CONVERGENCE}

In the following we shall attempt to validate the accuracy and general performance of the proposed scheme for several different multilayer two-dimensional optical elements. When exact solutions are available, we shall compare our results with exact solutions. However, for more interesting cases, these are not available, and we compare against independently verified highly accurate solutions obtained with a spectral multidomain timedomain code. ${ }^{5,6}$

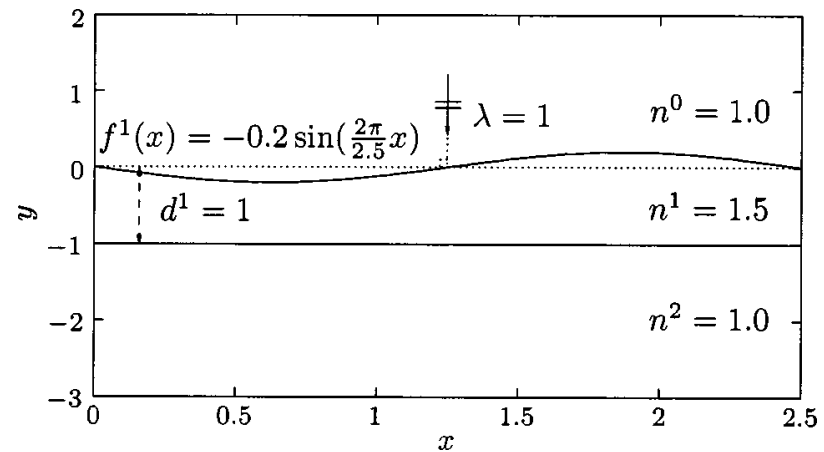

Fig. 5. Problem specification for a single layer with a shallow curved interface.

\section{A. Plane Interfaces}

In the case where all interfaces are planar, the solution is known in analytic form ${ }^{16}$ as

$$
u^{j}=A_{1}^{j} \exp \left[i\left(\alpha_{1}^{j} x-\beta_{1}^{j} y\right)\right]+A_{2}^{j} \exp \left[i\left(\alpha_{2}^{j} x+\beta_{2}^{j} y\right)\right]
$$

within each layer, assuming that the stack of layers is illuminated by a plane wave $A \exp [i(\alpha x-\beta y)]$. The unknown amplitudes are found by connecting the solutions 
through the boundary conditions (2) and (3) with $A_{1}^{0}$ $=A_{2}^{N}=0$. For the wave directions we have $\alpha_{1}^{j}=\alpha_{2}^{j}$ $=\alpha,|\alpha|+\left|\beta_{1}^{j}\right|=\left|k^{j+1}\right|$, and $|\alpha|+\left|\beta_{2}^{j}\right|=\left|k^{j}\right|$. This forms a linear system, which can be solved, producing $A_{l}^{j}$ for $j=0, \ldots, N$ and $l=1,2$. We can then compare the amplitude of the solution given by the exact solution, $\left|A_{l}^{j}\right|$, with the amplitude of the solution given by the multilayer boundary variation scheme, $\left|B_{l}^{j}\right|$, for $j=0, \ldots, N$ and $l$ $=1,2$ as a function of bounces.

\section{Thin Plate}

For the first test we consider a normal TE wave of unit amplitude illuminating a thin plate (see Fig. 3). We use Fresnel's equations to solve the single-interface problems and take the wave amplitude threshold $w_{\epsilon}=10^{-15}$; i.e., all waves are allowed to live for a maximum number of bounces.

In Fig. 3 we see that the solution, or rather the amplitudes, converges exponentially fast in the number of

Table 1. Convergence of Scattering Efficiencies and Relation to the Threshold Value $w_{\epsilon}$ Used in the Iterative Approach

\begin{tabular}{cccccccc}
\hline$w_{\epsilon}$ & $e_{0}^{+}$ & $e_{1}^{+}$ & $e_{2}^{+}$ & $e_{0}^{-}$ & $e_{1}^{-}$ & $e_{2}^{-}$ & Total Efficiency -1 \\
\hline $10^{-2}$ & 0.0175 & 0.0443 & 0.0032 & 0.7082 & 0.0814 & 0.0018 & $-1.30 \times 10^{-3}$ \\
$10^{-4}$ & 0.0167 & 0.0458 & 0.0057 & 0.7108 & 0.0828 & 0.0019 & $-5.34 \times 10^{-5}$ \\
$10^{-6}$ & 0.0167 & 0.0458 & 0.0057 & 0.7110 & 0.0828 & 0.0019 & $4.84 \times 10^{-6}$ \\
$10^{-8}$ & 0.0167 & 0.0458 & 0.0057 & 0.7110 & 0.0828 & 0.0019 & $-1.64 \times 10^{-8}$ \\
$10^{-10}$ & 0.0167 & 0.0458 & 0.0057 & 0.7110 & 0.0828 & 0.0019 & $-2.98 \times 10^{-10}$ \\
$10^{-12}$ & 0.0167 & 0.0458 & 0.0057 & 0.7110 & 0.0828 & 0.0019 & $3.81 \times 10^{-12}$ \\
$10^{-14}$ & 0.0167 & 0.0458 & 0.0057 & 0.7110 & 0.0828 & 0.0019 & $-1.54 \times 10^{-14}$ \\
$10^{-16}$ & 0.0167 & 0.0458 & 0.0057 & 0.7110 & 0.0828 & 0.0019 & $-2.22 \times 10^{-16}$ \\
$10^{-18}$ & 0.0167 & 0.0458 & 0.0057 & 0.7110 & 0.0828 & 0.0019 & $6.66 \times 10^{-16}$ \\
\hline
\end{tabular}

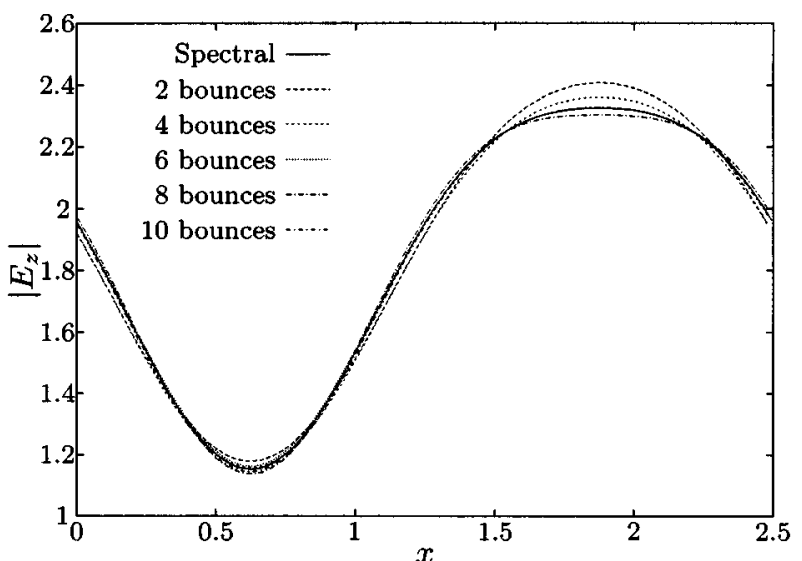

(a)

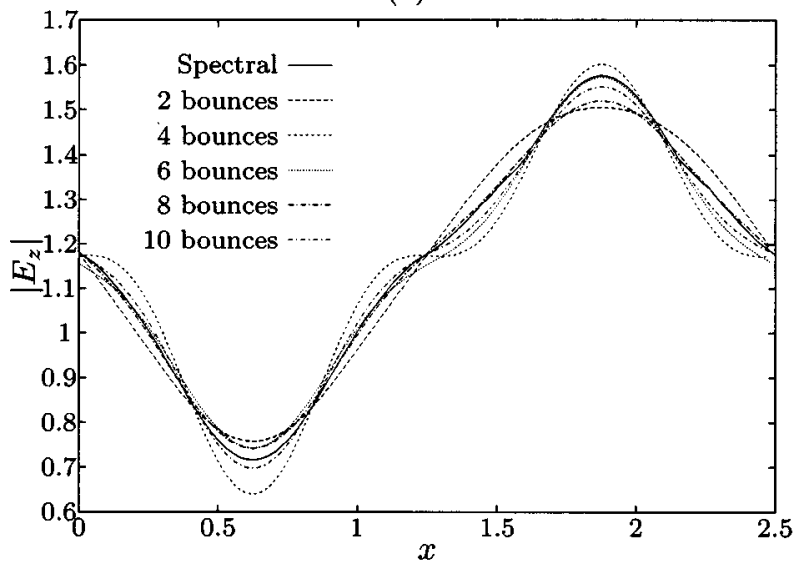

(c)

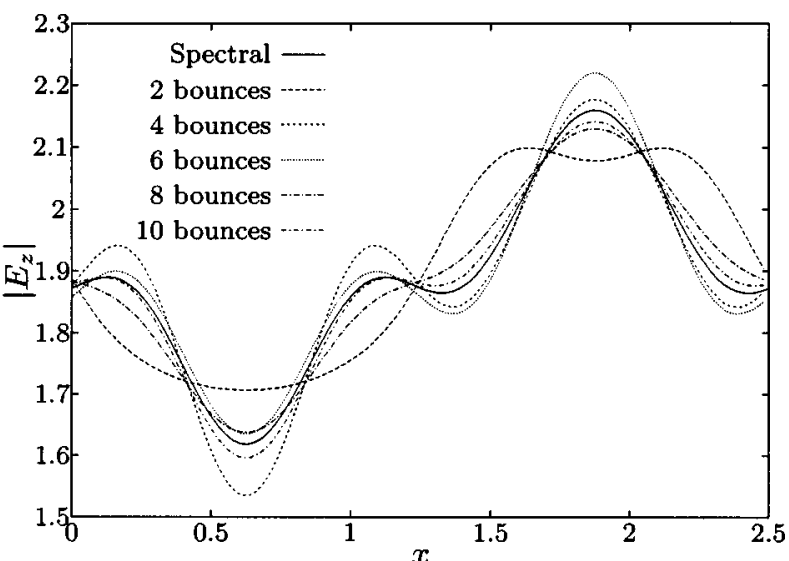

(b)

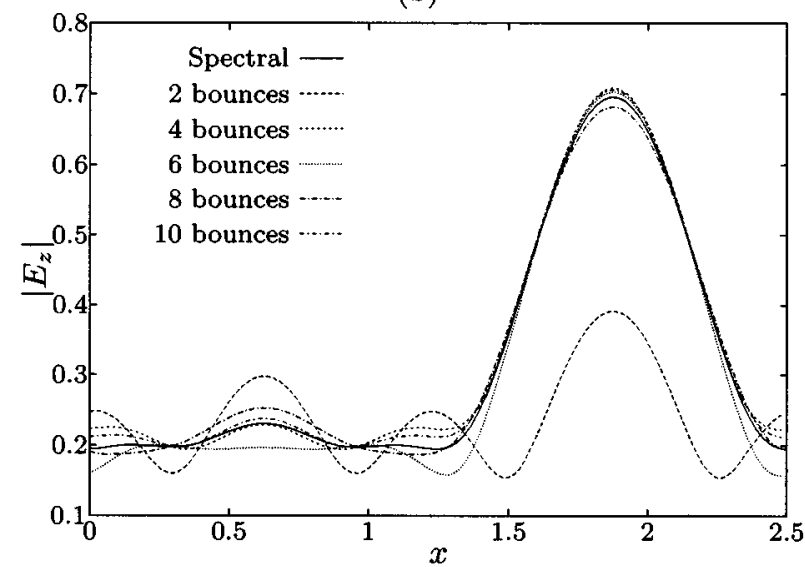

(d)

Fig. 6. $E_{z}$ computed at different heights $y$, with $y=0$ corresponding to the vertical position of the shallow curved interface: (a) $y$ $=-3$, (b) $y=-1$, (c) $y=-0.5$, (d) $y=3$. Illumination is TE polarized at normal incidence. Results are shown for a fixed number of internal bounces and compared with a highly accurate spectral solution, illustrating the importance of accounting for the multiple internal reflections. 


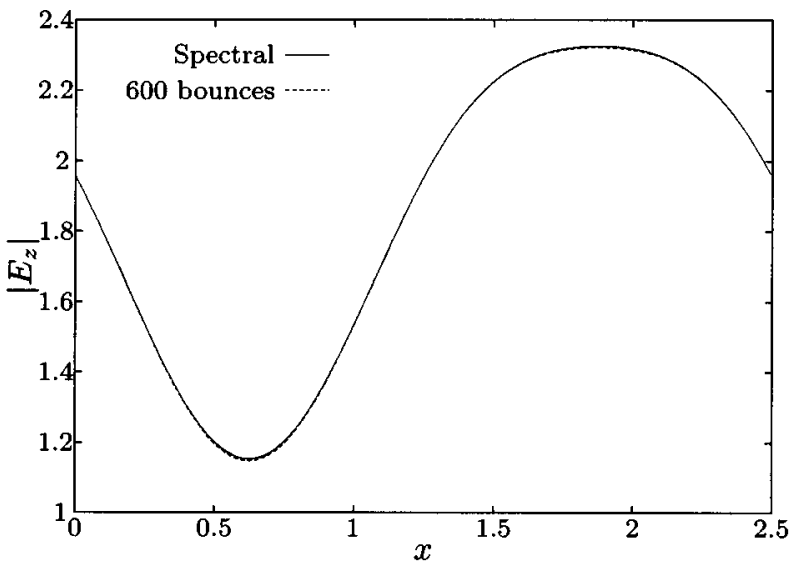

(a)

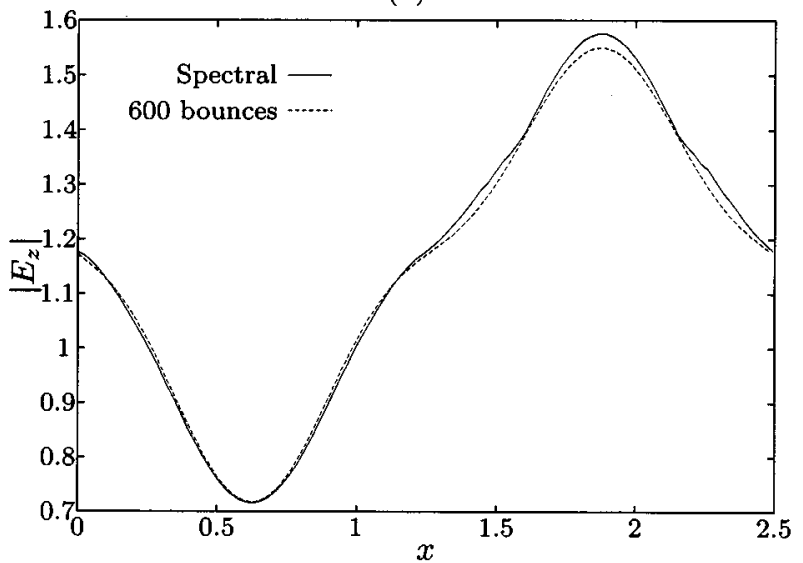

(c)

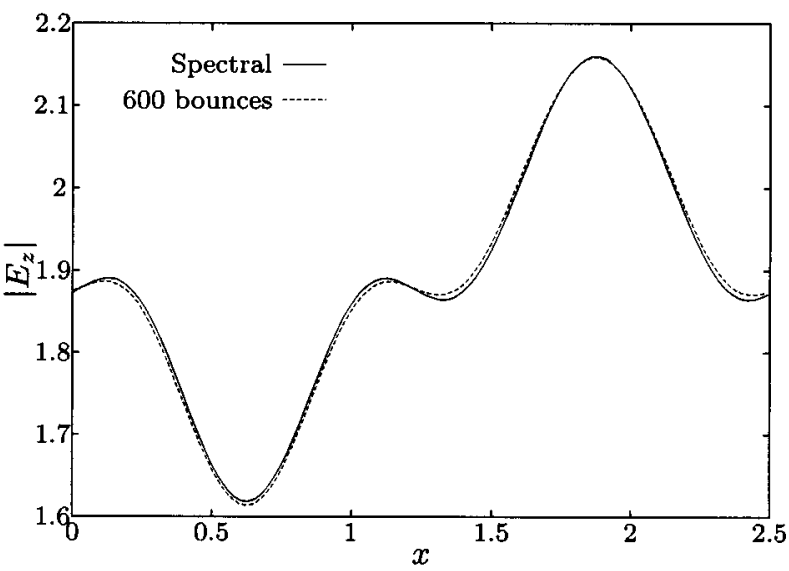

(b)

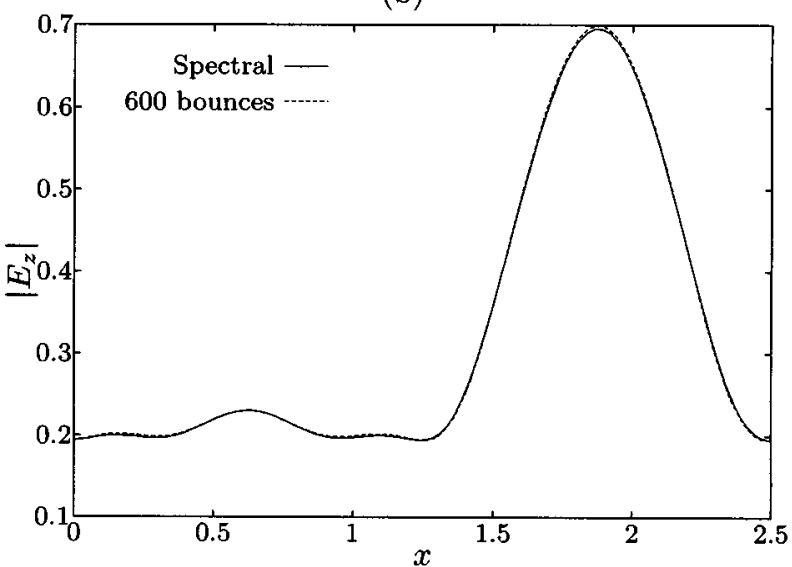

(d)

Fig. 7. $E_{z}$ computed at different heights $y$, with $y=0$ corresponding to the vertical position of the shallow curved interface: (a) $y$ $=-3$, (b) $y=-1$, (c) $y=-0.5$, (d) $y=3$. Illumination is TE polarized at normal incidence. Results are shown for converged solutions in terms of internal reflections and compared with a highly accurate spectral solution.

bounces to the exact solution. When comparing the solutions, we look at the error in complex amplitude of the waves in the exact solution $A_{l}^{j}$ with the computed solution $B_{l}^{j}$. As we increase the (maximum) number of bounces, i.e., $T^{\mathrm{ttl}}$, beyond 22 , the error remains constant. This is because the wave amplitude threshold $w_{\epsilon}$ is now fully responsible for terminating the waves.

\section{Thin Plate Stack}

A further test of accuracy is illustrated in Fig. 4, reflecting a normal TE wave of unit amplitude illuminating four thin plates stacked on top of each other. Again we use Fresnel's equations to solve the single-interface problems, since all of the interfaces are planar, and we choose the wave threshold to be $w_{\epsilon}=10^{-15}$.

This problem provides a test of collecting the waves being propagated into the set $W^{m+1}$ into a finite number of waves independent of number of bounces $m$. The work to compute the solution increases linearly with the number of bounces because of this collection process.

It is clear from Fig. 4 that there is exponentially fast convergence to the exact solution as the number of internal reflections increases. We can compute down to machine precision with 90 bounces in less than two tenths of a second on an average desktop computer. For bounces greater than 90 , the wave tolerance $w_{\epsilon}$ determines when the waves stop propagating.

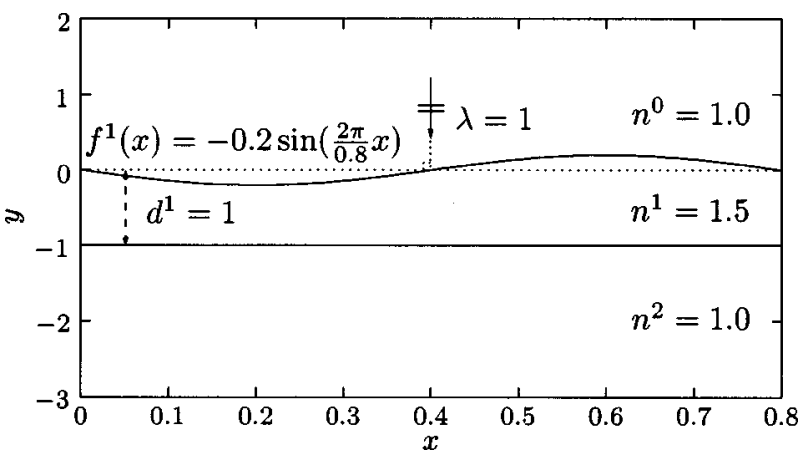

Fig. 8. Problem specification for a single layer with a deep curved interface.

\section{B. Single Curved Interfaces}

In the following we provide a few tests in which one interface is curved while all others remain planar. For simplicity, and supported by the general verifications in the above, we just consider the double-interface case. All results are compared with fully converged results obtained with a high-order accurate full field solver. ${ }^{5,6}$

\section{Shallow Single Curve}

This problem consists of a normal TE wave of unit amplitude illuminating a thin plate with one sinusoidal interface and a flat bottom, as illustrated in Fig. 5. The 
single-interface interactions with the sinusoidal interface $\Gamma^{1}$ are calculated by using a [31/31] Padé approximant and $E_{e}=0$. The interactions with $\Gamma^{2}$ are calculated by using Fresnel's equations.

The height-to-period ratio of $\Gamma^{1}$ is small (0.16), providing a simple test of the multilayer boundary variation code. While the efficiency table is being built, the singleinterface boundary variation scheme is used eight times to compute possible wave interactions with $\Gamma^{1}$ and the maximum error in the energy efficiency for the singleinterface cases is $1.33 \times 10^{-15}$.

Table 1 shows that the energy conservation from the single-interface scheme is kept as the number of bounces increases, i.e., there does not appear to be severe problems of error accumulation. In this table we increased the number of allowed internal bounces sufficiently to ensure that the wave tolerance $w_{\epsilon}$ is the factor that controls when the waves stop propagating.

To check the overall accuracy of the scheme, we illustrate in Figs. 6 and 7 the solutions obtained from the multilayer code with results obtained in a different manner. We see in Fig. 6 the importance of considering the multiple internal reflections. In Fig. 7 we have increased the number of internal reflections to ensure that waves

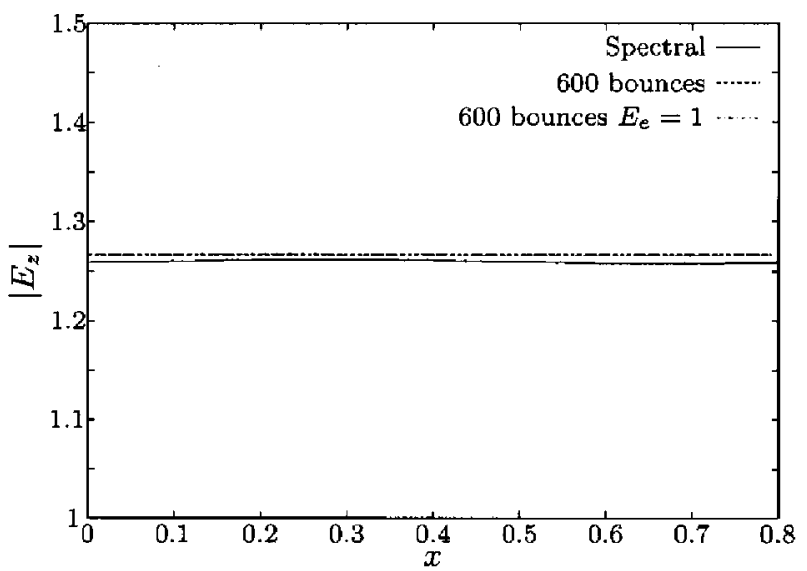

(a)

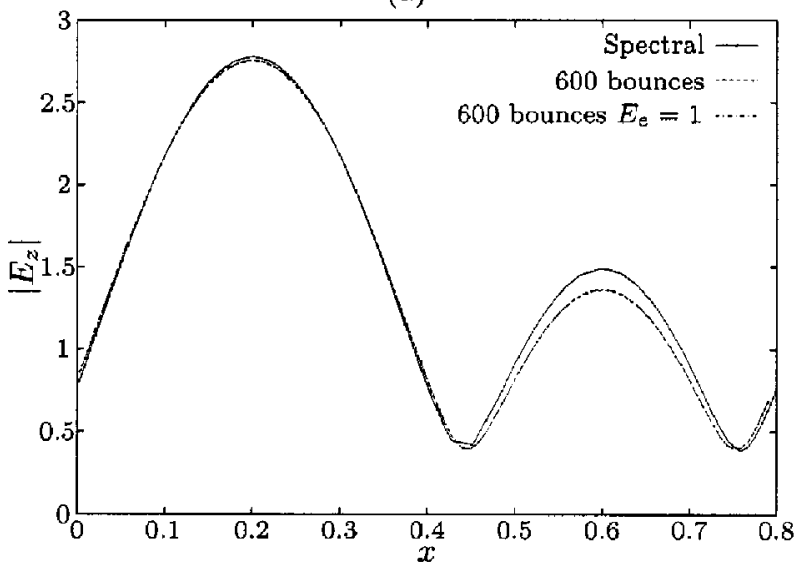

(c) are propagated until their amplitude is below $w_{\epsilon}$ $=10^{-15}$, yielding excellent agreement with the reference solution.

As one could expect, the multilayered boundary variation approximation becomes more accurate as the distance from the structure increases. This is a consequence of not including the evanescent waves that remain in the reference solution. The excellent agreement of the fields at different values of $y$ confirms that the phases of the fields are correct to a similar level of accuracy.

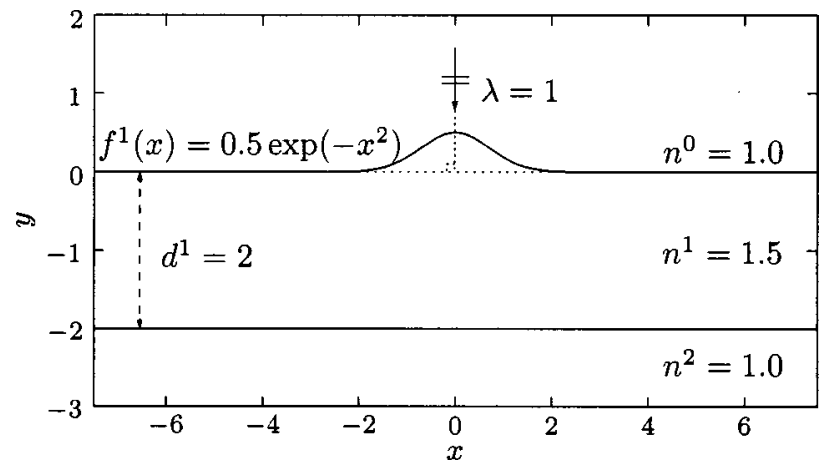

Fig. 10. Problem specification for a single layer with an integrated lens.

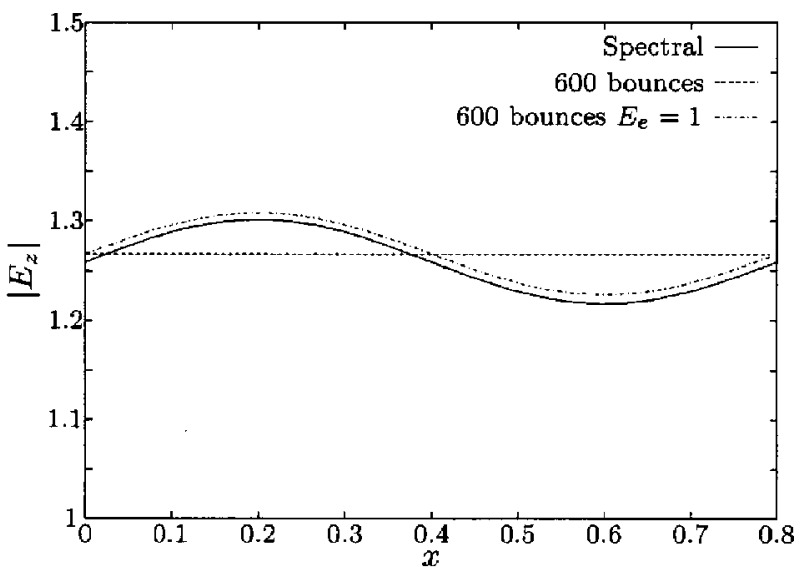

(b)

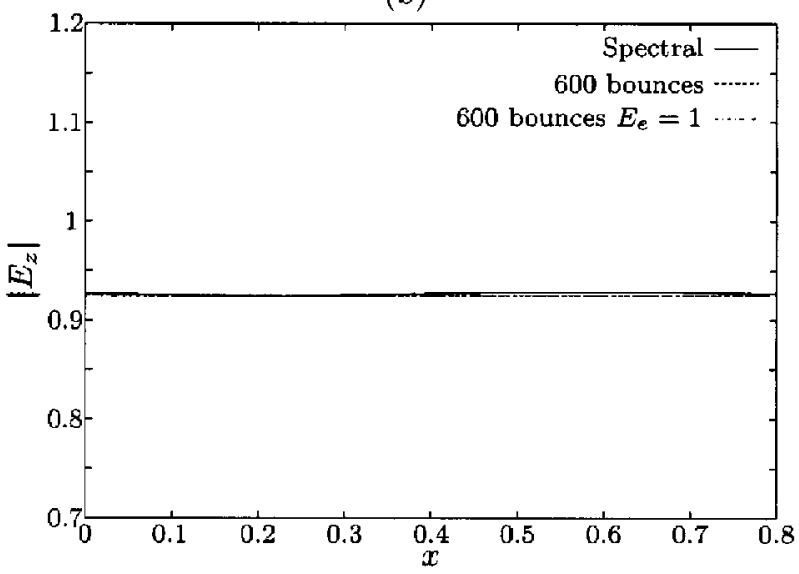

(d)

Fig. 9. $E_{z}$ computed at different heights $y$, with $y=0$ corresponding to the vertical position of the deep curved interface: (a) $y$ $=-3$, (b) $y=-1.5$, (c) $y=-0.5$, (d) $y=3$. Illumination is TE polarized at normal incidence. Results are shown for converged solutions in terms of internal reflections and compared with a highly accurate spectral solution. $E_{e}=1$ is a solution that includes the two evanescent modes from each single-interface solve with the smallest $|\beta|$. 


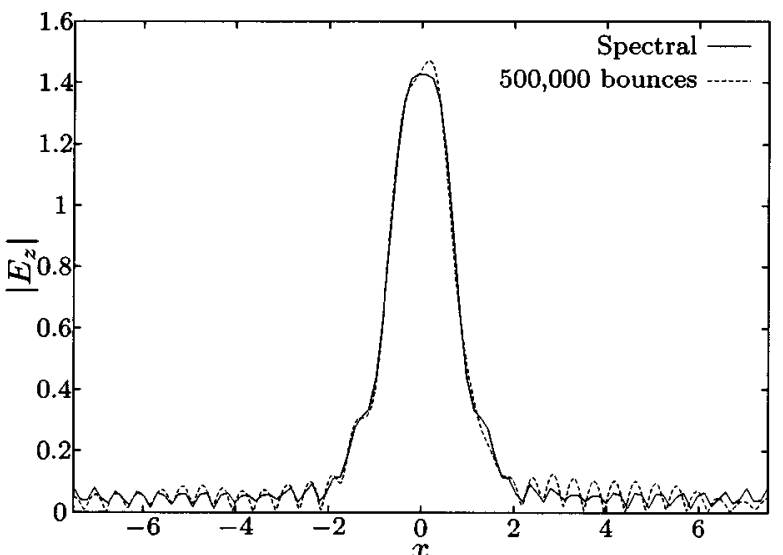

(a)

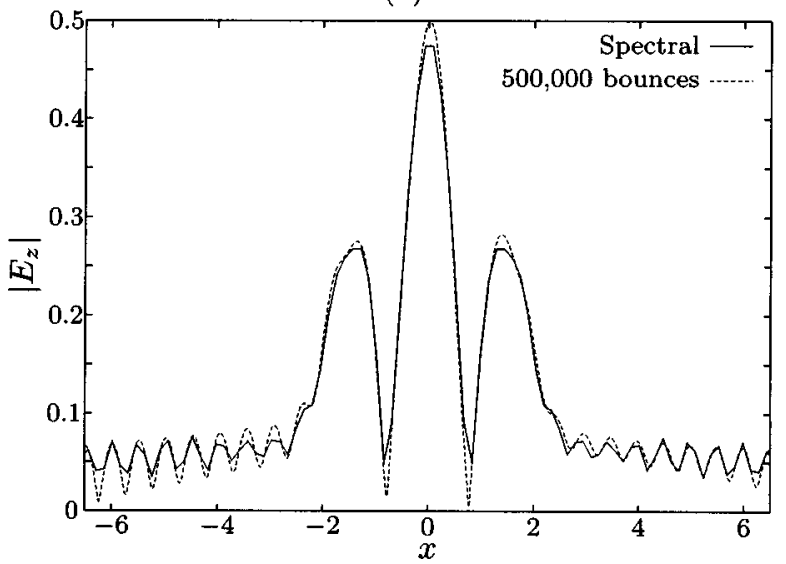

(c)

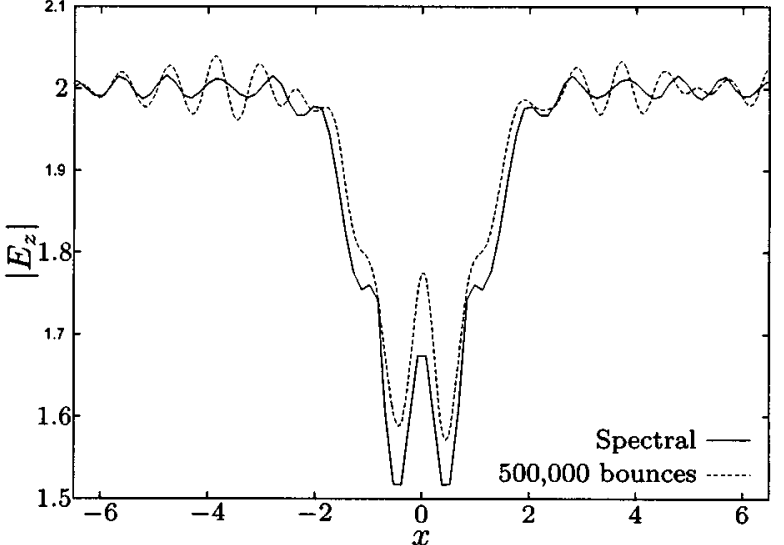

(b)

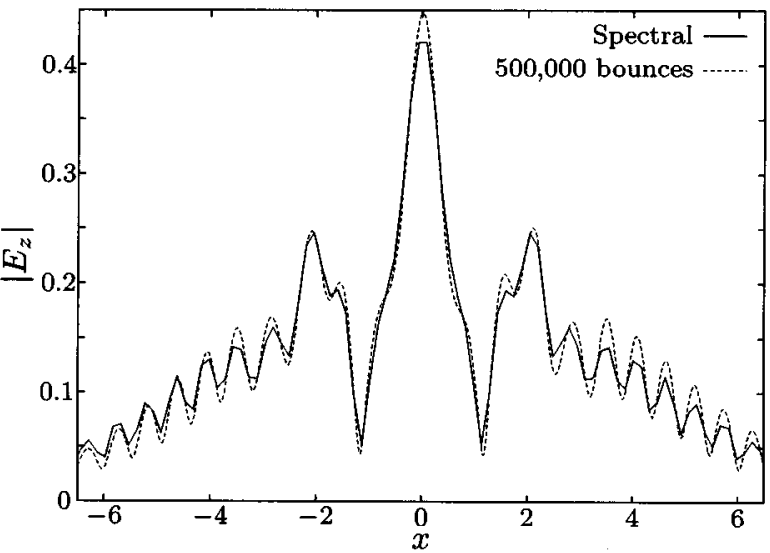

(d)

Fig. 11. $E_{z}$ computed at different heights $y$, with $y=0$ corresponding to the vertical position of the integrated lens: (a) $y=-2$, (b) $y=-1$, (c) $y=1$, (d) $y=3$. Illumination is TE polarized at normal incidence. Results are shown for converged solutions in terms of internal reflections and compared with a highly accurate spectral solution.

\section{Deeper Single Curve}

To further test the algorithm, we consider the case of a TE-polarized wave illuminating a double-interface problem in which the top interface varies considerably more than in the above case (see Fig. 8). In this case the height-to-period ratio of $\Gamma^{1}$ is 0.5 . The single-interface interactions with $\Gamma^{1}$ are calculated by using a [32/32] Padé approximant and $E_{e}=0$, leading to a maximum error in the efficiency for the single-interface cases of $\mathcal{O}\left(10^{-7}\right)$.

The total energy conservation is $\mathcal{O}\left(10^{-6}\right)$. Thus, as in the above simpler case, we find that the energy conservation of the multilayer boundary variation scheme is largely controlled by the maximum error in the efficiency for the single-interface cases.

In Fig. 9 we have increased the bounces enough so that all waves are propagated until the amplitude is below $w_{\epsilon}=10^{-7}$. The agreement with the reference solution is good and improves as one moves away from the structure. Also in the figure we present a solution that includes the two dominating evanescent modes from each singleinterface solve with the smallest $|\beta|$, and we denote this case as $E_{e}=1$. These waves are not propagated but are used in the solution. This provides a first-order correction, and we can see in Fig. 9(b), where $y=-1.5$, that this simple correction for the evanescent waves improves the solution. The evanescent modes that are included with $E_{e}>1$ are too small to change the plots given for $E_{e}=1$, so they are not presented. From the Bragg condition and energy conversation, we know that the amount at which the evanescent waves get damped increases exponentially as the order increases. Not accounting for the multiple reflections of the evanescent waves is the source for the remaining error in Fig. 9(c), where we are looking inside the layer.

\section{Lens}

As an example of a more general nonperiodic problem, we consider a Gaussian lens being illuminated by a normal TE wave of unit amplitude. The setup is illustrated in Fig. 10. Here $f^{1}(x)=0.5 \exp \left(-x^{2}\right)$ for $x \in(-7.5,7.5]$ and periodically extended. The periodicity of $\Gamma^{1}$ is set large enough so that its effect on the solution is small, thus approximating a nonperiodic surface. For the Fourier transform of $\Gamma^{1}$, needed by the single-interface boundary variation scheme, we use 33 modes, and the solutions are calculated by using a [37,37] Padé approximant with $E_{e}=0$. Since $\Gamma^{2}$ is flat, Fresnel's equations are used to calculate the scattering and penetration.

The multilayer boundary variation solution in Fig. 11 is for a sufficiently high number of internal reflections to ensure convergence, i.e., it is given for $w_{\epsilon}=10^{-15}$. In this 
case the error in efficiency is 0.8. However, the comparison with the direct solution reveals excellent agreement, in particular away from the lens.

\section{Double Curved Interfaces}

As a final test to illustrate the versatility of the developed scheme, we consider a TE wave of unit amplitude illuminating a thin structure with two curved interfaces, illustrated in Fig. 12. In this case both $\Gamma^{1}$ and $\Gamma^{2}$ are sinusoidal, and a [31/31] Padé approximant with $E_{e}=0$ is

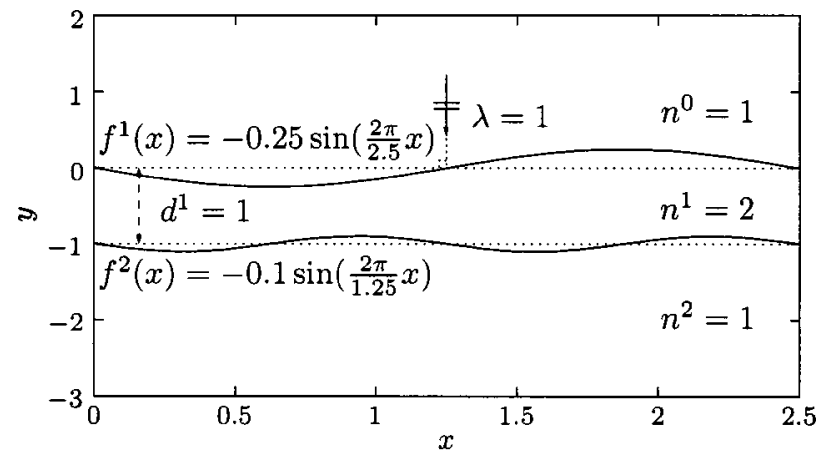

Fig. 12. Problem specification for a single layer with two curved interfaces.

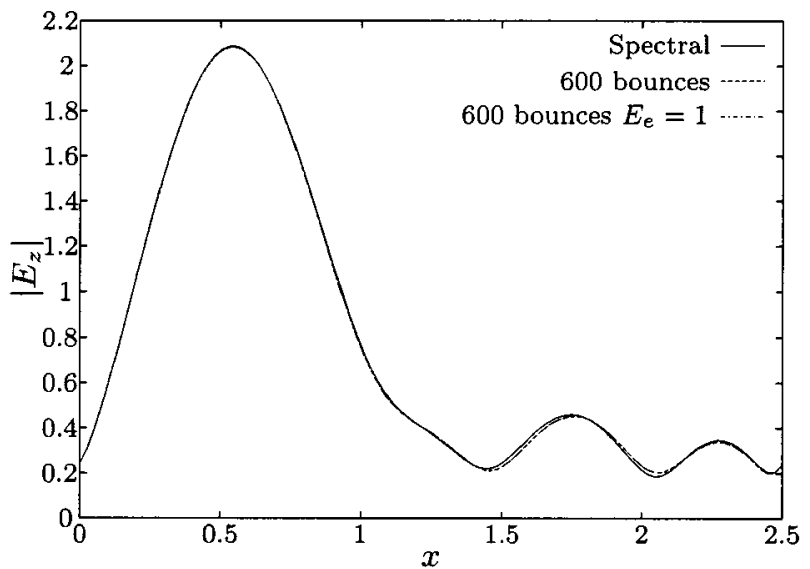

(a)

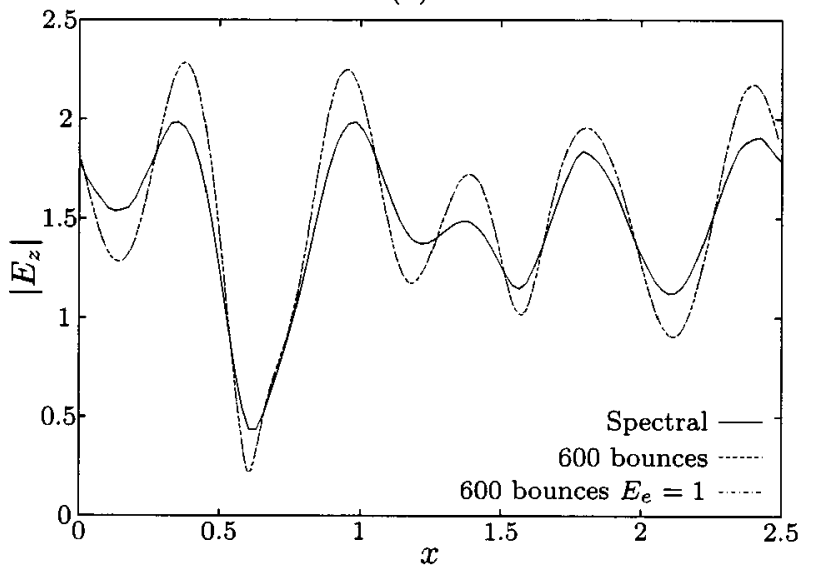

(c) used to calculate the single-interface interactions. The number of internal reflections is sufficiently high to ensure that all waves are propagated until their amplitude is below $w_{\epsilon}=10^{-15}$.

The results, shown in Fig. 12, show excellent agreement between the multilayer boundary variation scheme and the directly computed reference solution, in particular in the far-field region. The only exception is $y$ $=-0.5$. This can be attributed to the strong evanescent waves produced by the two curved interfaces and the high contrast. As we did in Section 2, we also include in Fig. 13 a solution that includes two evanescent modes from each single-interface solve $\left(E_{e}=1\right)$. In Fig. 13(b), where $y=-1.5$, we see that the correction for the evanescent waves improves the solution substantially. Here the error in efficiency is $\mathcal{O}\left(10^{-13}\right)$.

\section{E. Efficiency and Timings}

Table 2 lists the efficiency table size, the number of single-interface solves, and the approximate run times for the nonplanar cases presented above. As we can see, the time spent bouncing the waves is a small fraction of the total run time in each case; in particular, a large fraction of the computation time is spent in the preprocessing stage. The preprocessing stage is where the single-

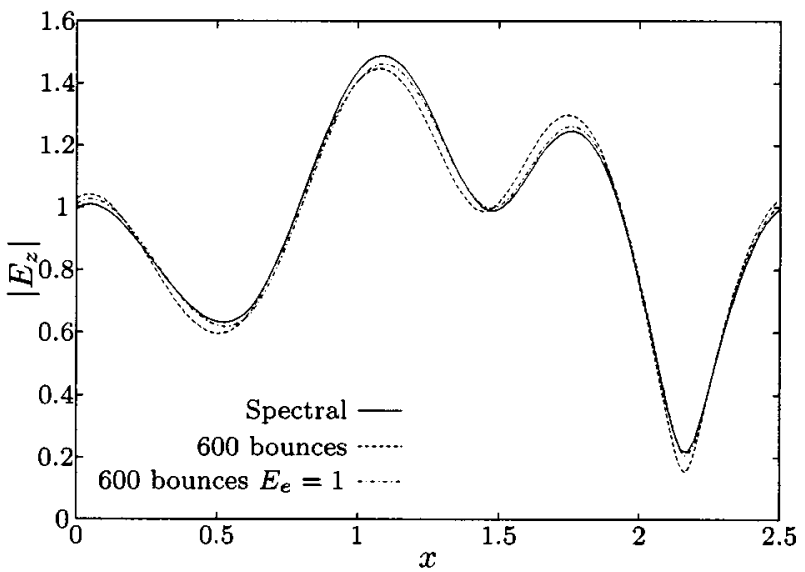

(b)

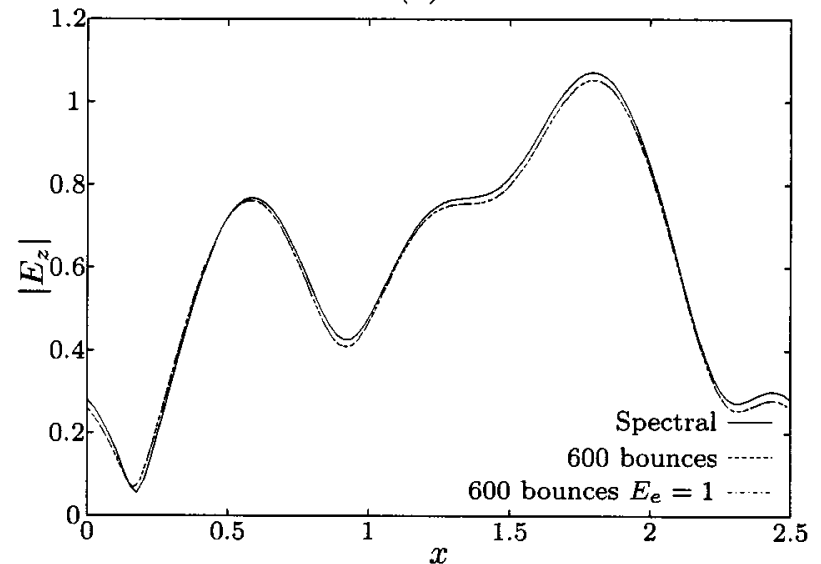

(d)

Fig. 13. $E_{z}$ computed at different heights $y$, with $y=0$ corresponding to the vertical position of the slowly varying interface: (a) $y$ $=-3$, (b) $y=-1.5$, (c) $y=-0.5$, (d) $y=3$. Illumination is TE polarized at normal incidence. Results are shown for converged solutions in terms of internal reflections and compared with a highly accurate spectral solution. $E_{e}=1$ is a solution that includes the two evanescent modes from each single-interface solve with the smallest $|\beta|$. 


\section{Table 2. Time and Space Used in the Multilayer Boundary Variation Method Computations Presented in the Paper}

\begin{tabular}{rcccc}
\hline Figure & $\begin{array}{c}\text { Number of } \\
\text { Single- } \\
\text { Interface } \\
\text { Solves }\end{array}$ & $\begin{array}{c}\text { Efficiency } \\
\text { Table Size } \\
\text { (number of } \\
\text { waves) }\end{array}$ & $\begin{array}{c}\text { Approximate } \\
\text { Time to } \\
\text { Bounce Waves } \\
(\mathrm{s})^{\mathrm{a}}\end{array}$ & $\begin{array}{c}\text { Approximate } \\
\text { Total Run } \\
\text { Times } \\
(\min )^{\mathrm{a}}\end{array}$ \\
\hline 5 & 8 & 15 & 0.13 & 0.5 \\
8 & 4 & 7 & 0.08 & 0.25 \\
10 & 49 & 101 & 466 & 1230 \\
12 & 19 & 19 & 0.13 & 1.1 \\
\hline
\end{tabular}

${ }^{a}$ All times are approximate wall clock time for the computations on an 800-MHz AMD Athlon processor.

interface solutions are computed for waves in all directions supported by the problem. We also note that the lens case (Fig. 10) takes longer than the rest for a couple of reasons. The single-interface solutions are harder to compute because the interface requires 33 Fourier modes to be represented instead of three for the other cases. Also, the larger period of the lens allows, through the Bragg condition, more propagating modes, increasing the workload. All times are approximate wall clock time for the computations on an $800-\mathrm{MHz}$ AMD Athlon processor.

\section{CONCLUDING REMARKS}

The main purpose of this work has been to develop an efficient and accurate computational approach to model multilayered optical elements, with periodic profiles at each interface separating layers of homogeneous nonmagnetic magnetic materials. If the far field is of main interest, aperiodic interfaces can be modeled by using periodic interfaces and near-to-far-field transformations. ${ }^{11}$

At the heart of the scheme is a very efficient boundary variation method for accurately solving the problem of reflection and refraction by a single interface, be it material or metallic. The method exploits the linearity of the Helmholtz equation, along with the Bragg condition, to significantly reduce the amount of space and time needed to compute the solutions consisting of numerous internally reflected waves. A detailed understanding of the scattering processes allows us to collect the waves into a finite set of active waves, limiting the otherwise exponentially growing set of waves. Following a setup phase, tens of thousands of internally reflected waves can subsequently be computed at very little additional cost.

In the cases presented here, the evanescent waves produced by the single-interface boundary variation scheme are often disregarded, as we have focused on the solution away from the grating structure. However, for other applications, one could include these in a way similar to that used for propagating waves. Given that the kernel is the single-interface scheme, the limitation is set by this; i.e., in double-precision arithmetic the height-to-period ratio of the variations of the interfaces cannot exceed $\mathcal{O}(1)$ without encountering numerical stability problems. Using extended precision can help this, although the cost typically increases.
While these results offer the first step in the development of a general high-order accurate method for the efficient modeling of multilayered diffractive optics, a number of important issues remain open. Straightforward extensions include illumination by Gaussian beams, by solving a sequence of problems subject to plane-wave illumination, and the use of the threshold $w_{\epsilon}$ to adaptively control the work and the requested accuracy. Extensions to the three-dimensional vectorial case is likewise straightforward, following the past developments of appropriate solutions for one interface. ${ }^{10,12}$

\section{ACKNOWLEDGMENTS}

The work of L. C. Wilcox was supported by the National Science Foundation (NSF) through a Vertical Integration of Research and Education in the Mathematical Sciences program at Brown University. The work of J. S. Hesthaven was partially supported by the U.S. Army Research Office under contract DAAD19-01-1-0631, by the NSF through an NSF Career Award, and by the Alfred P. Sloan Foundation through a Sloan Research Fellowship.

Address correspondence to Jan.Hesthaven@Brown.edu.

\section{REFERENCES}

1. T. K. Gaylord and M. G. Moharam, "Analysis and applications of optical diffraction by gratings," Proc. IEEE 73, 894937 (1985).

2. B. Lichtenberg and N. C. Gallagher, "Numerical modeling of diffractive devices using the finite element method," Opt. Eng. 33, 1592-1598 (1994).

3. K. Hirayama, E. N. Glytsis, T. K. Gaylord, and D. W. Wilson, "Rigorous electromagnetic analysis of diffractive cylindrical lenses," J. Opt. Soc. Am. A 13, 2219-2231 (1996)

4. D. W. Prather and S. Shi, "Formulation and application of the finite-difference time-domain method for the analysis of axially symmetric diffractive optical elements," J. Opt. Soc. Am. A 16, 1131-1141 (1999).

5. P. G. Dinesen, J. S. Hesthaven, J. P. Lynov, and L. Lading, "Pseudospectral method for the analysis of diffractive optical elements," J. Opt. Soc. Am. A 16, 1124-1130 (1999).

6. J. S. Hesthaven, P. G. Dinesen, and J. P. Lynov, "Spectral collocation time-domain modeling of diffractive optical elements," J. Comput. Phys. 155, 287-306 (1999).

7. J. S. Hesthaven, "High-order accurate methods in timedomain computational electromagnetics: a review," Adv. Electron. Electron Phys. 127, 59-123 (2003).

8. O. Bruno and F. Reitich, "Numerical solution of diffraction problems: a method of variation of boundaries," J. Opt. Soc. Am. A 10, 1168-1175 (1993).

9. O. Bruno and F. Reitich, "Numerical solution of diffraction problems: a method of variation of boundaries. II. Finitely conducting gratings, Padé approximants, and singularities," J. Opt. Soc. Am. A 10, 2307-2316 (1993).

10. O. Bruno and F. Reitich, "Numerical solution of diffraction problems: a method of variation of boundaries. III. Doubly periodic gratings,” J. Opt. Soc. Am. A 10, 2551-2562 (1993)

11. P. G. Dinesen and J. S. Hesthaven, "A fast and accurate boundary variation method for diffractive gratings," J. Opt. Soc. Am. A 17, 1565-1572 (2000)

12. P. G. Dinesen and J. S. Hesthaven, "A fast and accurate boundary variation method for diffractive gratings. II. 
The three-dimensional vectorial case," J. Opt. Soc. Am. A 18, 2876-2885 (2001).

13. R. Petit, "A tutorial introduction," in Electromagnetic Theory of Gratings, R. Petit, ed., Vol. 22 of Topics in Current Physics (Springer-Verlag, Berlin, 1980), pp. 1-52.

14. O. Bruno and F. Reitich, "Solution of a boundary value problem for Helmholtz equation via variation of the bound- ary into the complex domain," Proc. R. Soc. Edinburgh, Sect. A 122, 317-340 (1992)

15. G. A. Baker and P. Graves-Morris, Padé Approximants, 2nd ed., Vol. 59 of Encyclopedia of Mathematics and Its Applications (Cambridge U. Press, Cambridge, UK. 1996).

16. P. Yeh, Optical Waves in Layered Media (Wiley, New York, 1988). 